\title{
Coil Intrabronchial Migration in an Arteriovenous Malformation Patient Treated 10 Years Ago
}

\author{
Tadashi Umehara, MD, Masaya Aoki, MD, PhD, Go Kamimura, MD, Kazuhiro Wakida, MD, \\ Toshiyuki Nagata, MD, Tsunayuki Otsuka, MD, and Masami Sato, MD, PhD
}

\begin{abstract}
A 56-year-old male who had received transcatheter coil deposition 10 years ago for an arteriovenous malformation (AVM) was admitted to our hospital because of persistent hemosputum. Chest radiograph and bronchoscopy revealed straightened coil bundles in his air way. Recently, less invasive transcatheter intervention has been performed more frequently for treatment of AVM than surgical resection. In our case, however, chest radiography and bronchoscopy showed that the coils might migrate from the deposited site to the airway. Then, right lower lobectomy was undertaken. In AVM patient who received coil deposition, a long-term follow-up is recommended, and surgical resection should be carried out if necessary.
\end{abstract}

Keywords: arteriovenous malformation, endovascular procedures, coil migration,

surgical resection

\section{Introduction}

Recently, less invasive transcatheter intervention has been performed more frequently for treatment of arteriovenous malformation (AVM) than surgical resection. We report a case whose deposited coils migrated into the large air way 10 years after coil embolization.

\section{Case Report}

A 56-year-old male was admitted to our hospital because of persistent bloody sputum. The patient had a history of smoking (1 pack per day for 34 years) and

Department of General Thoracic Surgery, Graduate School of Medical and Dental Sciences, Kagoshima University, Kagoshima, Kagoshima, Japan

Received: October 18, 2016; Accepted: November 20, 2016 Corresponding author: Masami Sato, MD, PhD. Department of General Thoracic Surgery, Graduate School of Medical and Dental Sciences, Kagoshima University, 8-35-1 Sakuragaoka, Kagoshima, Kagoshima 890-8520, Japan

Email: m-sato@m2.kufm.kagoshima-u.ac.jp

(C)2017 The Editorial Committee of Annals of Thoracic and Cardiovascular Surgery. All rights reserved. repeated nasal bleeding since adolescence. At 46 years of age, the patient underwent transcatheter coil embolization in another hospital for treatment of an AVM. Interlocking detachable coils (IDC18: $2 \mathrm{~mm} \times 4 \mathrm{~cm}$ and IDC18 Soft: $2 \mathrm{~mm} \times 2 \mathrm{~cm}$, Boston Scientific, Marlborough, MA, USA) were used for the operation. Moreover, when the patient was 56 years old, he suffered from a brain abscess and meningitis. His father also had a history of brain abscess with AVM.

Six months before admission to our hospital, the patient received bronchial artery embolization for sudden hemoptysis, but this persisted. The chest radiograph showed coil deposits in the AVM in the right lower lobe. Some of the coils migrated into the right main bronchus (Fig. 1a, arrow 1) and the lung parenchyma with infiltrative shadows (Fig. 1a, arrow 2). The migrated "coils" were straight.

Bronchoscopic examination revealed the proximal ends of the "coil" bundles were in the trachea (Fig. 1b). Transcatheter bronchial artery embolization was repeated but failed. So, surgical resection was performed.

During the operation, the right basal bronchus was partially incised and the migrated coils, which were located more proximally in the bronchus, were pulled 


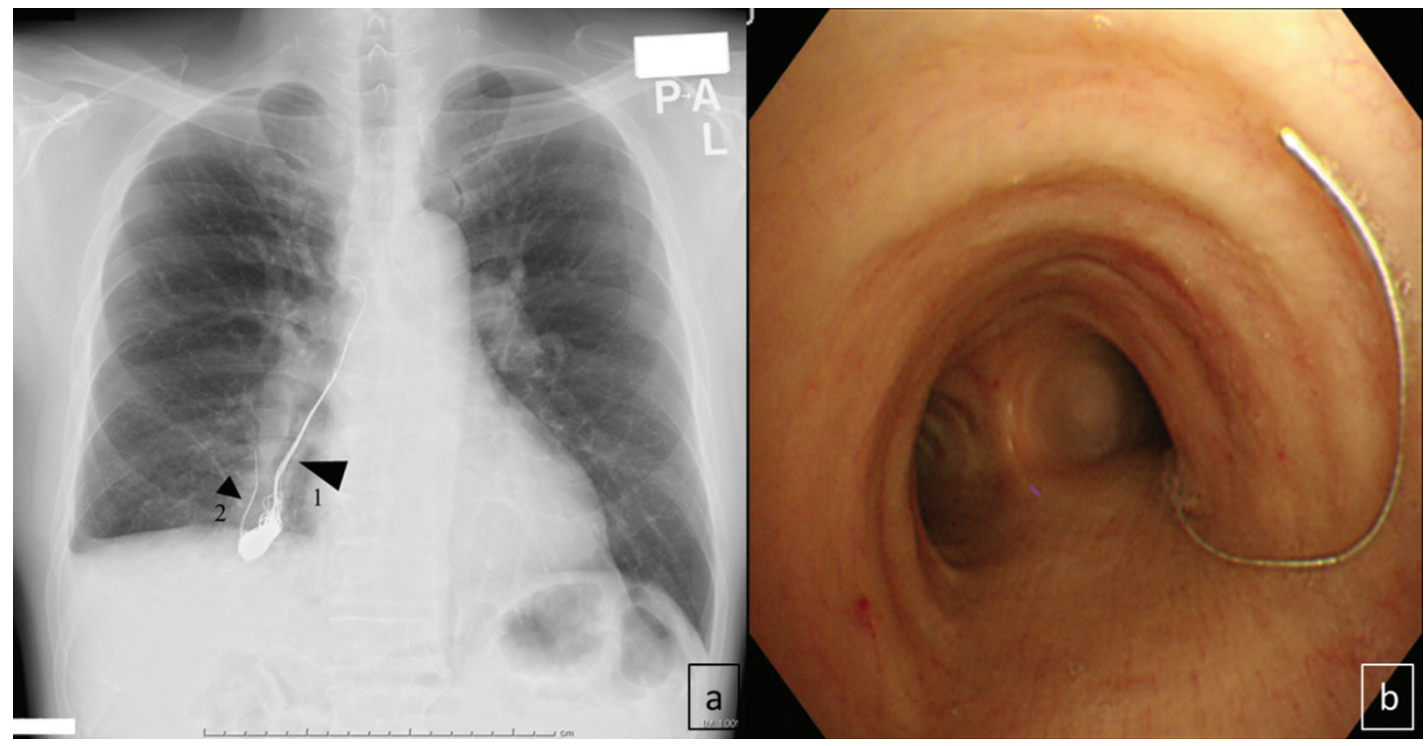

Fig. 1 Chest radiograph on admission (a) and bronchoscopic findings (b). (a) Straightened "coil" bundles are seen both in the right main bronchus (arrow 1) from the AVM and in the lung parenchyma (arrow 2). (b) Proximal end of coils were presented in the trachea. No active bleeding was observed. AVM: arteriovenous malformation

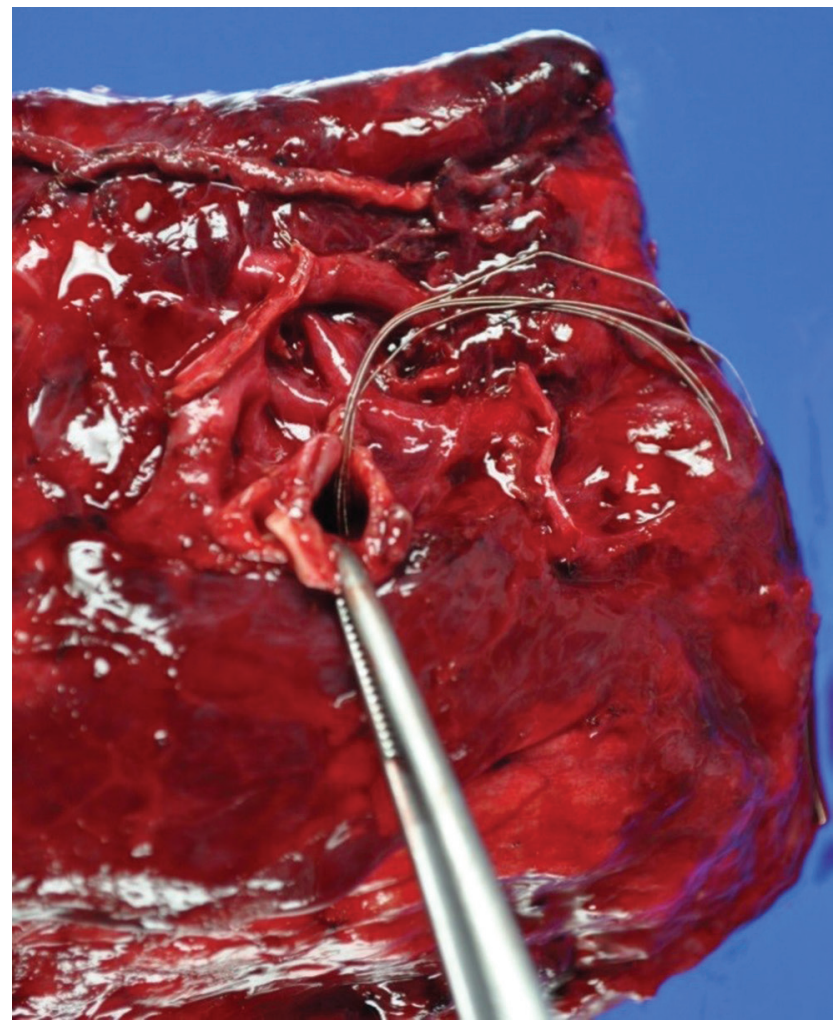

Fig. 2 Macroscopic view of the resected specimen. "Coil" bundles were detected from the bronchial orifice. The "coils" had been straightened.

out. After the A6-A10 and inferior pulmonary vein were ligated, the lower lobe bronchus, which was more proximal than the incision site, was cut using a surgical stapler
(Endo GIA Black, Medtronic, Dublin, Ireland) (Fig. 2). The postoperative course was uneventful; the patient was discharged 2 weeks later and has been well for 2 years, till date.

\section{Discussion}

AVM is a pulmonary vascular disorder characterized by abnormal communications between pulmonary artery and vein without a microcapillary network. More than $80 \%$ of AVM are congenital and $47 \%-80 \%$ of those are associated with hereditary hemorrhagic telangiectasia in Western countries. ${ }^{1)}$ The risk of AVM rupture increases with diameter. ${ }^{2)}$

In 26\%-33\% of patients who did not receive any treatment for AVM, brain infarction, brain abscess, or rapture of the lesion were reported. ${ }^{3)}$

Indications for AVM treatment are hypoxia, heart failure, or central nervous system complication(s). Even in asymptomatic cases, intensive treatment is recommended when the influx artery diameter is more than $3 \mathrm{~mm}$ or the AVM diameter is more than $20 \mathrm{~mm}^{2)}$ There are some reports that transcatheter embolization is useful for these patients instead of surgical resection. ${ }^{4)}$

From our knowledge of follow-up data of patients who underwent transcatheter embolization for pulmonary vascular disorders, there are few reports that deal with recanalization or increase in AVM diameter. 
Only one pulmonary artery aneurysm case with coil migration has been reported in a patient who underwent lobectomy, in whom coil migration was observed 6 weeks after coil deposition. ${ }^{5}$ ) Weakness of the arterial wall, erosion of the adjacent bronchus, and failure of coils to maintain its spiral shape were pointed out as the mechanisms of the coil migration.

Our case is unique for two reasons: 1) It is the first case of coil migration in an AVM patient and 2) coil migration occurred after such prolonged coil placement as 10 years.

Although the actual mechanisms of coil migration in our case are still unknown, one possible explanation may be the shape of the deposited coils. At the time of hemoptysis, the coil was straight rather than coiled. Based on this, we speculate that the end of the linear coil, which caused migration and hemoptysis, compressed the surrounding tissues for long periods of time.

Compared with systemic arteries, pulmonary vessels have thinner walls. Thus, long-term follow-up should be recommended in cases with pulmonary vessel embolization, especially when the deposited coils are straight.
Attention is necessary because future surgical care may be required in some cases.

\section{Disclosure Statement}

The authors have nothing to disclose with regard to commercial support.

\section{References}

1) Vase P, Holm M, Arendrup H. Pulmonary arteriovenous fistulas in hereditary hemorrhagic telangiectasia. Acta Med Scand 1985; 218: 105-9.

2) Gossage JR, Kanj G. Pulmonary arteriovenous malformations. A state of the art review. Am J Respir Crit Care Med 1998; 158: 643-61.

3) Burke CM, Safai C, Nelson DP, et al. Pulmonary arteriovenous malformations: a critical update. Am Rev Respir Dis 1986; 134: 334-9.

4) Pollak JS, Saluja S, Thabet A, et al. Clinical and anatomic outcomes after embolotherapy of pulmonary arteriovenous malformations. J Vasc Interv Radiol 2006; 17: 35-44; quiz 45.

5) Abad J, Villar R, Parga G, et al. Bronchial migration of pulmonary arterial coil. Cardiovasc Intervent Radiol 1990; 13: 345-6. 